\title{
Acumulación de forraje de pasto buffel e híbridos de Urochloa a diferente edad de rebrote
}

\section{Forage accumulation of buffelgrass and Urochloa hybrids at different regrowth ages}

\author{
Jonathan R. Garay-Martínez ${ }^{1}$, Santiago Joaquín-Cancino ${ }^{1 *}$, Benigno Estrada-Drouaillet ${ }^{1}$, Juan C. \\ Martínez-González ${ }^{1}$, Bertín M. Joaquín-Torres ${ }^{2}$, Andrés G. Limas-Martínez ${ }^{1}$, Javier Hernández-Meléndez ${ }^{1}$. \\ ${ }^{1}$ Facultad de Ingeniería y Ciencias, Universidad Autónoma de Tamaulipas. Centro Universitario Campus Victoria, Edificio \\ Centro de Gestión del Conocimiento $4^{\circ}$ piso. CP. 87120. Ciudad Victoria, Tamaulipas, México. \\ ${ }^{2}$ Universidad del Papaloapan, Campus Loma Bonita. Departamento de Zootecnia. Av. Ferrocarril s/n, Col. Ciudad \\ Universitaria. CP. 68400. Loma Bonita, Oaxaca. México. \\ ${ }^{*}$ Autor de correspondencia: sjoaquin@docentes.uat.edu.mx
}

Nota científica recibido: 12 de septiembre de 2017 aceptado: 14 de abril de 2018

RESUMEN. Se evaluaron diferentes cultivares de Urochloa (Insurgente, Cayman, Cobra y Mulato II) y pasto Buffel H-17 (como testigo) a diferente edad de rebrote (ER), durante la época de máxima (EMAP) y mínima precipitación (EMIP). Las variables evaluadas fueron materia seca total (MST), hoja (MSh) y tallo (MSt) y área foliar específica (AFE). Durante la EMAP, Cayman y Mulato II presentaron la mayor MST ( $9 \mathrm{t} \mathrm{MS} \mathrm{ha}^{-1}$ ); mientras que en EMIP el testigo registró el menor valor (1.04 vs. $0.79 \mathrm{t} \mathrm{MS} \mathrm{ha}^{-1}$ ). Durante EMAP Mulato II presentó mayor MSh (8.40 t MS $\mathrm{ha}^{-1}$ ). La MSt se incrementó conforme aumentó la ER. Cayman y Mulato Il registraron los valores más altos de AFE $\left(199 \mathrm{~cm}^{2} \mathrm{~g}^{-1}\right)$. Independientemente de la época, la MST, MSh y MSt se incrementaron conforme se aumentó la ER. Cayman y Mulato II son los cultivares más promisorios por su alta acumulación de forraje, MSh y AFE.

Palabras clave: Brachiaria híbrido CIAT 36087, Brachiaria híbrido BR02/1752, Brachiaria híbrido BR02/1794, Pennisetum ciliare $\mathrm{H}-17$

ABSTRACT. Different Urochloa cultivars (Insurgente, Cayman, Cobra and Mulato II) and H-17 bufffelgrass (as control) were evaluated at different regrowth ages (RA) during the maximum and minimum precipitation periods (MAPP and MIPP, respectively). The evaluated variables were total, leaf and stem dry matter (TDM, LDM and SDM, respectively) and specific leaf area (SLA). During the MAPP, Cayman and Mulato II had the highest TDM (9 t DM $\mathrm{ha}^{-1}$ ), while in the MIPP the control recorded the lowest value (1.04 vs. $\left.0.79 \mathrm{t} \mathrm{DM} \mathrm{ha}^{-1}\right)$. During the MAPP, Mulato II had the highest LDM (8.40 t DM ha $\left.{ }^{-1}\right)$. SDM increased as the RA increased. Cayman and Mulato II recorded the highest SLA values (199 $\mathrm{cm}^{2} \mathrm{~g}^{-1}$, average). Regardless of the period, the TDM, LDM and SDM increased as the RA increased. Cayman and Mulato II are the most promising cultivars due to their high forage accumulation, LDM and SLA.

Key words: Brachiaria hybrid CIAT 36087, Brachiaria hybrid BR02/1752, Brachiaria hybrid BR02/1794, Pennisetum ciliare $\mathrm{H}-17$

\section{INTRODUCCIÓN}

En el estado de Tamaulipas, México, 4.67 millones de hectáreas están dedicadas a la ganadería, de esta superficie el $78 \%$ corresponde a agostaderos y el $22 \%$ restante a praderas con pastos nativos o introducidos (SAGARPA 2010) como pas- to Bermuda (Cynodon dactylon L.) y pasto Buffel (Pennisetum ciliare L.) (Gómez et al. 2007), con rendimientos de hasta $18 \mathrm{t} \mathrm{MS} \mathrm{ha}{ }^{-1}$ año ${ }^{-1}$, bajo condiciones de riego (SIAP 2014). En este contexto, el $27 \%$ del territorio estatal corresponde a climas secos, donde el periodo de lluvias se presenta en los meses de mayo a octubre (Vargas et al. 2007). 


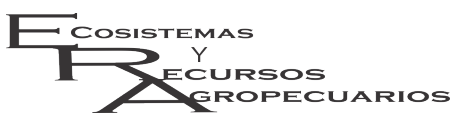

Actualmente, existen nuevos cultivares de gramíneas forrajeras que superan en rendimiento de materia seca a los pastos nativos. Los cultivares de Urochloa híbrido (sin. Brachiaria híbrido): Cobra y Cayman, presentan rendimientos de 9 a 14 t MS ha $^{-1}$ año $^{-1}$ (Pizarro et al. 2013). Estos cultivares además de tolerar la sequía (Arroyave et al. 2013) pueden crecer en suelos alcalinos y ácidos (MoralesBautista et al. 2011). Por ello, dichos cultivares pueden adaptarse a las condiciones del clima y suelos del estado de Tamaulipas, con el objetivo de incrementar la productividad animal.

La capacidad productiva de cualquier especie forrajera se determina por su composición genética (Miles 2006), edad de la planta, condiciones climáticas presentes a través del año (Lara et al. 2010, Garay et al. 2017b) y manejo agronómico (CruzHernández et al. 2017), los cuales modifican las características morfológicas y estructurales de la planta (Dourado et al. 2015). Se ha mencionado que el conocimiento de la influencia de la estacionalidad en el crecimiento y producción de forraje en las especies de interés para una determinada región es importante, ya que permite conocer el tiempo de mayor y menor disponibilidad de forraje y adoptar estrategias de manejo para maximizar la producción animal; por ello, se debe evaluar el efecto estacional y la dinámica de crecimiento de nuevos cultivares de gramíneas forrajeras para optimizar su aprovechamiento (Avellaneda et al. 2008). Se ha señalado que antes de introducir una especie de gramínea forrajera, a cualquier sistema de producción, se debe evaluar su comportamiento forrajero (Maass et al. 2015). Por tanto, el objetivo del presente estudio fue evaluar la dinámica de rendimiento, acumulación y distribución de materia seca en función de la edad de rebrote en diferentes cultivares del pasto Urochloa y Pennisetum ciliare cv. H-17, bajo condiciones de temporal y, con ello, determinar si dichos cultivares son una opción para la ganadería de la región.
Garay-Martínez et al. Comportamiento forrajero de pasto Urochloa Ecosist. Recur. Agropec. 5(15):573-581,2018

\section{MATERIALES Y MÉTODOS}

\section{Localización del estudio y características edafo- climáticas}

El estudio se realizó en condiciones de temporal, de mayo 2016 a junio 2017 en la Posta Zootécnica Ingeniero Herminio García González de la Facultad de Ingeniería y Ciencias de la Universidad Autónoma de Tamaulipas. Las coordenadas geográficas son $23^{\circ} 56^{\prime} 26.5^{\prime \prime} \mathrm{N}$ y $99^{\circ} 05^{\prime} 59.9^{\prime \prime} \mathrm{O}$, a 193 metros sobre el nivel del mar (INEGI 2015). El clima del lugar es de tipo BS1 ( $h$ ') hw (Vargas et al. 2007). La temperatura y precipitación media anual es de $24.1^{\circ} \mathrm{C}$ y $940 \mathrm{~mm}$, respectivamente, ocurriendo la mayor precipitación de mayo a octubre (CONAGUA 2010). La precipitación y temperatura registradas durante el periodo experimental se presentan en la Figura 1. El suelo del lugar es de textura arcillosa $(11.3,23.3$ y $65.4 \%$, arena, limo y arcilla, respectivamente), con $\mathrm{pH}$ de 8.3 , la relación de adsorción de sodio es de 0.19 , materia orgánica de $4.2 \%, 0.25 \%$ de $\mathrm{N}, 7.4,288.6,1.4$ y $0.46 \mathrm{mg}$ $\mathrm{kg}^{-1}$ de $\mathrm{P}, \mathrm{K}, \mathrm{Fe}$ y $\mathrm{Zn}$, respectivamente (Garay et al. 2017a).

\section{Tratamientos y diseño experimental}

Se evaluaron cuatro cultivares de Urochloa ( $U$. brizantha cv. Insurgente y $U$. híbrido cvs. Cayman, Mulato II y Cobra) y Pennisetum ciliare cv. H17, como testigo. Los tratamientos se distribuyeron en un diseño completamente al azar, con cuatro repeticiones, en arreglo de parcelas divididas, donde la parcela grande fue la edad de rebrote $(4,6$ y 8 semanas) y la parcela chica, los cinco cultivares. El tamaño de las parcelas experimentales fue de 25 $\mathrm{m}^{2}$, con una parcela útil de $1 \mathrm{~m}^{2}$.

\section{Establecimiento y manejo de la pradera}

La siembra se realizó al voleo el 16 de octubre de 2015 empleando semilla comercial para todos los cultivares, a dosis de siembra de $4 \mathrm{~kg} \mathrm{ha}^{-1}$ de semilla pura viable. Se consideró un periodo de establecimiento de siete meses, durante el cual se realizaron dos cortes de uniformidad a $15 \mathrm{~cm}$ sobre el suelo, para homogenizar las praderas. Después 


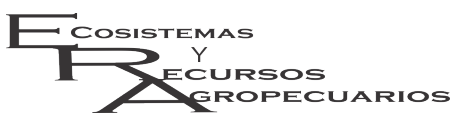

Garay-Martínez et al.

Comportamiento forrajero de pasto Urochloa

Ecosist. Recur. Agropec.

5(15):573-581,2018

del segundo corte de uniformidad, 27 de mayo de 2016, se inició la evaluación. Al momento de la siembra se fertilizó al voleo, con $120,60,70$, y 50 $\mathrm{kg} \mathrm{ha}^{-1}$ de $\mathrm{N}, \mathrm{P}_{2} \mathrm{O}_{5}, \mathrm{~K}_{2} \mathrm{O}$, y $\mathrm{SO}_{4}$, respectivamente. Las fuentes de fertilizantes fueron: Urea, Sulfato de amonio, Fosfato monoamónico y Cloruro de potasio. El nitrógeno, se fraccionó en tres aplicaciones: durante la siembra, a los cuatro y ocho meses posteriores.

\section{Variables evaluadas}

Se evaluó el rendimiento, la acumulación de materia seca total (MST), hoja (MSh) y tallo (MSt) y área foliar específica (AFE). Las evaluaciones se realizaron a las 4,6 y 8 semanas, cosechando periódicamente el forraje presente en $1 \mathrm{~m}^{2}$. El forraje cosechado se pesó y se tomó una submuestra de $200 \mathrm{~g}$, la cual se separó en sus componentes morfológicos: hoja (vaina + lámina foliar) y tallo. De cada submuestra se tomaron las láminas foliares de 10 tallos para determinar el área foliar específica $\left(\mathrm{cm}^{2} \mathrm{~g}^{-1}\right)$, utilizando un integrador de área modelo Cl-202 (CID Bio-Science). Posteriormente, las muestras se colocaron en una estufa de aire forzado a $65{ }^{\circ} \mathrm{C}$ por $48 \mathrm{~h}$. Después del periodo de secado, se registró el peso seco de las submuestras de forraje y se estimó el rendimiento y acumulación (t MS ha ${ }^{-1}$ ) de materia seca total, de hoja y tallo para cada edad de rebrote.

\section{Análisis estadístico}

Los datos de las variables evaluadas se analizaron con el procedimiento GLM de SAS (2004) con base en un diseño completamente al azar con arreglo de parcelas divididas. La comparación de medias de tratamientos se efectuó mediante la prueba de Tukey, con un nivel de significancia del $5 \%$.

\section{RESULTADOS Y DISCUSIÓN}

\section{Rendimiento de forraje}

La precipitación y temperatura registradas durante el periodo de evaluación (Figura 1) determinaron el rendimiento de forraje. En este sen- tido, durante el periodo de mayor precipitación (620 $\mathrm{mm}$ ) y temperatura (mayo-octubre), se obtuvo el mayor rendimiento de forraje, con valores que oscilaron de 0.18 a $3.04,0.85$ a 4.63 y de 1.10 a $5.71 \mathrm{t} \mathrm{ha}^{-1}$, para las 4,6 y 8 semanas, respectivamente. En este periodo también se observó disminución del rendimiento de forraje en los meses de julio y agosto, debido a la sequía intraestival ("Canícula"). Por otra parte, en el periodo de mínima precipitación (noviembre-abril, $148 \mathrm{~mm}$ ), se obtuvieron los menores rendimientos de forraje con valores de 0.03 a $0.35,0.08$ a 0.45 y de 0.10 a 0.64 t MS ha ${ }^{-1}$ para la cosecha de forraje a las 4, 6 y 8 semanas, respectivamente (Figura 1 ).

El alto rendimiento de forraje obtenido en la época de máxima precipitación se puede atribuir a la disponibilidad de humedad y temperatura, con lo que se favoreció el crecimiento de los cultivares (Cruz et al. 2011b, Cruz-Hernández et al. 2017). Por el contrario durante la época de mínima precipitación la escasa disponibilidad de agua y bajas temperaturas de hasta $3{ }^{\circ} \mathrm{C}$, limitaron el crecimiento y el rendimiento de forraje. Se ha indicado que la temperatura óptima para el crecimiento de los cultivares de Urochloa y Pennisetum ciliare oscila entre 13 y $42{ }^{\circ} \mathrm{C}$ (Durán et al. 2011); por tanto, cuando la temperatura desciende por debajo, o bien supera dicho rango, se interrumpen las funciones fisiológicas y metabólicas como la respiración y fotosíntesis, lo que inhibe el crecimiento de la planta (Cardoso et al. 2015).

\section{Acumulación de materia seca total (MST)}

Durante la época de mayor precipitación, se encontró diferencias $(\mathrm{p} \leq 0.05)$ en la acumulación de MST, donde los cultivares Cayman y Mulato II presentaron los mayores valores con 9.10 y 9.01 t MS $\mathrm{ha}^{-1}$, respectivamente (Tabla 1 ). A las 4 semanas de rebrote los cultivares Cayman y Mulato II tuvieron la mayor acumulación de forraje con 8.89 y $8.77 \mathrm{t} \mathrm{MS} \mathrm{ha}^{-1}$, respectivamente, seguido de $\mathrm{H}-17$ con $8.21 \mathrm{t} \mathrm{MS} \mathrm{ha}^{-1}$; mientras que la menor acumulación de MST fue para los cultivares Insurgente y Cobra, con 6.49 y 6.31 t MS ha $^{-1}$, respectivamente. Para las ocho semanas, los cultivares Cayman y Co- 


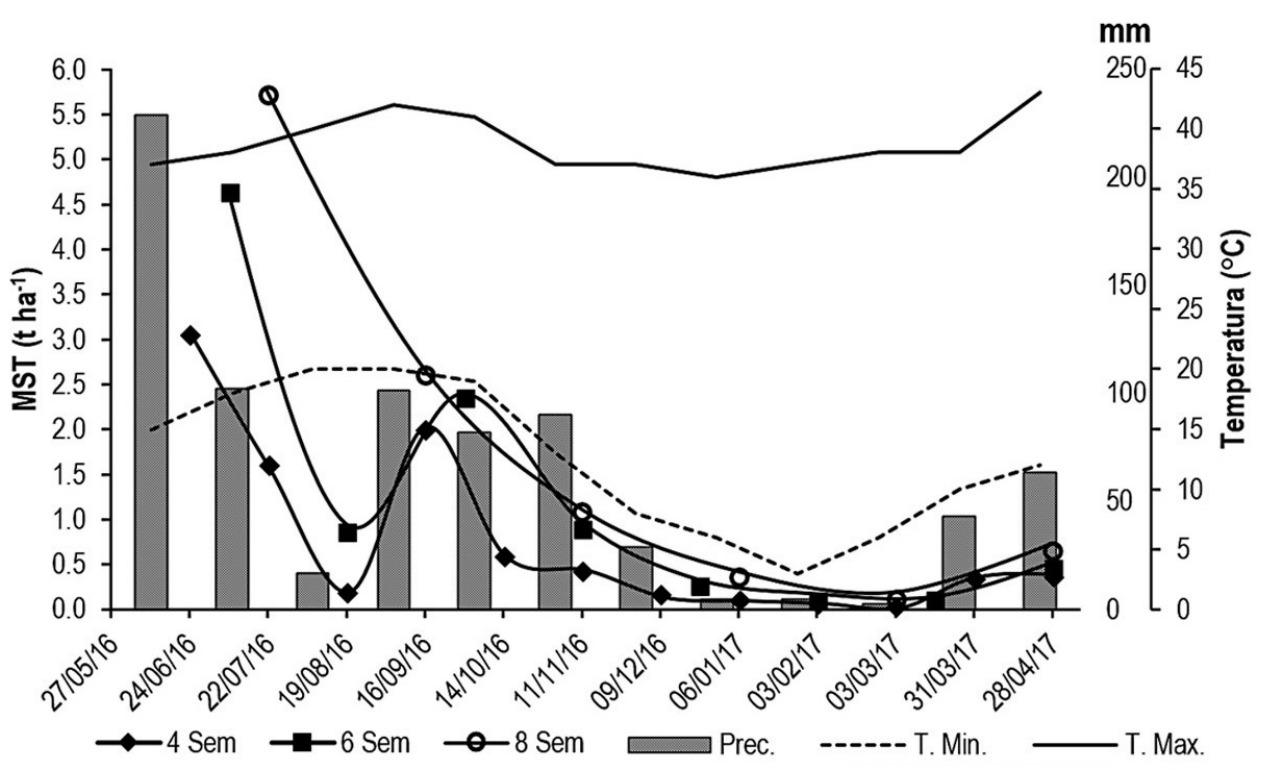

Figura 1. Rendimiento de materia seca total ( $\mathrm{t} M S$ ha ${ }^{-1}$ ) en las épocas de máxima (mayooctubre 2016) y mínima (noviembre 2016-abril 2017) precipitación $(\mathrm{mm})$, a diferente edad de rebrote (semanas). Sem $=$ Semanas; Prec $=$ Precipitación; $\mathrm{T}$. Min $=$ Temperatura Mínima; T. Max $=$ Temperatura Máxima.

bra presentaron la mayor acumulación de forraje con 9.50 y 10.13 t MS ha $^{-1}$ y H-17 la menor con $8.55 \mathrm{t}$ $\mathrm{MS} \mathrm{ha}^{-1}$. Durante la época de menor precipitación, todos los cultivares de Urochloa $(\mathrm{p} \leq 0.05)$ presentaron mayor acumulación de forraje (1.00 a 1.13 t MS ha ${ }^{-1}$ ), en comparación con el cultivar $\mathrm{H}-17$ $\left(0.79 \mathrm{t} \mathrm{MS} \mathrm{ha}^{-1}\right)$. A las 6 y 8 semanas de rebrote, el cultivar Cobra presentó la mayor acumulación con 1.04 y 1.36 t MS ha $^{-1}$, respectivamente (Tabla 1 ). Se observó que la acumulación de forraje se incrementó con forme se aumentó la edad de rebrote, con valores de 7.73 a 9.31 y de 0.88 a 1.13 t MS $\mathrm{ha}^{-1}$ para las 4 y 8 semanas, durante las épocas de máxima y mínima precipitación, respectivamente (Tabla 1).

La acumulación de MST obtenida en los cultivares de Urochloa fue similar a lo reportado por Pizarro et al. (2013), aun cuando el presente estudio se realizó en clima seco y suelo con $\mathrm{pH}$ alcalino; además, la acumulación de forraje que se obtuvo en los cultivares de Urochloa fue mayor al testigo Pennisetum ciliare (10 vs. $9 \mathrm{t} \mathrm{MS} \mathrm{ha}^{-1} \mathrm{año}^{-1}$ ). En el presente estudio, el rendimiento mayor de forraje obtenido en los cultivares de Urochloa, en comparación con Pennisetum ciliare cv. H-17 pudo deberse a las características intrínsecas de dichos cultivares, ya que según Pizarro et al. (2010), los híbridos Cobra y Cayman, son cultivares de segunda generación, con alto potencial para la producción de forraje. En cambio, el rendimiento menor de forraje en la edad de rebrote de 4 semanas es consecuencia del intervalo corto de la planta para la acumulación de reservas (Donaghy et al. 2008), lo cual se traduce en una menor tasa de crecimiento (Cruz-Hernández et al. 2017).

\section{Acumulación de materia seca de hoja (MSh)}

La acumulación de MSh varió entre cultivares y edad de rebrote $(p \leq 0.05)$. Durante la época de mayor precipitación el cultivar Mulato II presentó la mayor acumulación de hoja con promedio de $8.40 \mathrm{t}$ $\mathrm{MS} \mathrm{ha}^{-1}$, seguido de los cultivares Cayman e Insurgente con 7.74 y 7.25 t MS ha $^{-1}$, respectivamente; mientras que el cultivar $\mathrm{H}-17$ registró la menor acumulación con un valor de 6.21 t MS ha $^{-1}$ (Tabla 1). A las cuatro semanas después de rebrote los culti- 
Tabla 1. Acumulación de materia seca total (MST), hoja (MSh), tallo (MSt) y área foliar especifica (AFE) en cultivares de Urochloa spp. y Pennisetum ciliare, a diferente edad de rebrote, durante los periodos de máxima (mayo-octubre) y mínima (noviembre-abril) precipitación.

\begin{tabular}{|c|c|c|c|c|c|c|c|c|}
\hline \multirow{2}{*}{ Cultivar } & \multicolumn{3}{|c|}{ Edad de rebrote (semanas) } & \multirow{2}{*}{ Promedio } & \multicolumn{3}{|c|}{ Edad de rebrote (semanas) } & \multirow{2}{*}{ Promedio } \\
\hline & 4 & 6 & 8 & & 4 & 6 & 8 & \\
\hline Máxima precipitación & \multicolumn{4}{|c|}{ MST $(\mathrm{t} \mathrm{ha}-1$ ) } & \multicolumn{4}{|c|}{ MSh $\left(\mathrm{t} \mathrm{ha}^{-1}\right)$} \\
\hline Cayman & $8.89^{a *}$ & $8.91^{a}$ & $9.50^{a b}$ & $9.10^{a}$ & $8.44^{a *}$ & $8.00^{a}$ & $6.78^{b}$ & $7.74^{b}$ \\
\hline Cobra & $6.31^{c}$ & $8.19^{a}$ & $10.13^{a}$ & $8.21^{b}$ & $5.59^{d}$ & $6.37^{b}$ & $7.82^{a}$ & $6.59^{d}$ \\
\hline Mulato II & $8.77^{a b}$ & $9.08^{a}$ & $9.19^{b c}$ & $9.01^{a}$ & $8.49^{a}$ & $8.48^{a}$ & $8.23^{a}$ & $8.40^{a}$ \\
\hline Insurgente & $6.49^{c}$ & $8.22^{a}$ & $9.16^{b c}$ & $7.96^{b}$ & $6.23^{c}$ & $7.38^{a b}$ & $8.15^{a}$ & $7.25^{c}$ \\
\hline $\mathrm{H}-17$ & $8.21^{b}$ & $8.27^{a}$ & $8.55^{c}$ & $8.34^{b}$ & $6.80^{b}$ & $6.50^{b}$ & $5.33^{c}$ & $6.21^{d}$ \\
\hline \multirow[t]{2}{*}{ Promedio } & $7.73^{C}$ & $8.53^{B}$ & $9.31^{A}$ & & $7.11^{A}$ & $7.35^{A}$ & $7.26^{A}$ & \\
\hline & \multicolumn{4}{|c|}{ MSt $\left(\mathrm{t} \mathrm{ha}{ }^{-1}\right)$} & \multicolumn{4}{|c|}{$\operatorname{AFE}\left(\mathrm{g} \mathrm{cm}^{-2}\right)$} \\
\hline Cayman & $0.35^{b *}$ & $0.39^{b}$ & $0.88^{b c}$ & $0.54^{a}$ & $216^{a *}$ & $204^{a}$ & $202^{a}$ & $207^{a}$ \\
\hline Cobra & $0.37^{b}$ & $1.03^{a}$ & $1.24^{b}$ & $0.88^{a}$ & $196 b^{c}$ & $187^{b}$ & $189^{b}$ & $191^{b}$ \\
\hline Mulato II & $0.22^{b}$ & $0.46^{b}$ & $0.37^{c}$ & $0.35^{a}$ & $199^{b}$ & $189^{b}$ & $185^{b}$ & $191^{b}$ \\
\hline Insurgente & $0.14^{b}$ & $0.55^{b}$ & $0.55^{c}$ & $0.41^{a}$ & $188^{c}$ & $177^{c}$ & $174^{c}$ & $180^{c}$ \\
\hline $\mathrm{H}-17$ & $1.12^{a}$ & $1.23^{a}$ & $2.27^{a}$ & $1.54^{b}$ & $177^{d}$ & $161^{d}$ & $192^{b}$ & $176^{c}$ \\
\hline Promedio & $0.44^{C}$ & $0.73^{B}$ & $1.06^{A}$ & & $195^{A}$ & $184^{C}$ & $188^{B}$ & \\
\hline \multicolumn{9}{|l|}{ Mínima precipitación } \\
\hline & \multicolumn{4}{|c|}{$\operatorname{MST}\left(\mathrm{t} \mathrm{ha}^{-1}\right)$} & \multicolumn{4}{|c|}{$\operatorname{MSh}\left(\mathrm{t} \mathrm{ha}^{-1}\right)$} \\
\hline Cayman & $0.96^{a}$ & $0.97^{b}$ & $1.20^{b}$ & $1.04^{a}$ & $0.96^{a}$ & $0.97^{b}$ & $1.20^{b}$ & $1.04^{a}$ \\
\hline Cobra & $0.98^{a}$ & $1.04^{a}$ & $1.36^{a}$ & $1.13^{a}$ & $0.98^{a}$ & $0.88^{a}$ & $1.36^{a}$ & $1.08^{a}$ \\
\hline Mulato II & $0.99^{a}$ & $1.01^{a b}$ & $1.07^{c}$ & $1.02^{a}$ & $0.99^{a}$ & $1.01^{b}$ & $1.07^{c}$ & $1.02^{a}$ \\
\hline Insurgente & $0.92^{a}$ & $0.99^{a b}$ & $1.10^{c}$ & $1.00^{a}$ & $0.92^{a}$ & $0.99^{a}$ & $1.10^{c}$ & $1.00^{a}$ \\
\hline $\mathrm{H}-17$ & $0.57^{a}$ & $0.88^{c}$ & $0.92^{d}$ & $0.79^{b}$ & $0.57^{a}$ & $0.88^{b}$ & $0.92^{d}$ & $0.79^{b}$ \\
\hline \multirow[t]{2}{*}{ Promedio } & $0.88^{B}$ & $0.98^{B}$ & $1.13^{A}$ & & $0.88^{B}$ & $0.95^{B}$ & $1.13^{A}$ & \\
\hline & \multicolumn{4}{|c|}{ MSt $\left(\mathrm{t} \mathrm{ha} \mathrm{a}^{-1}\right)$} & \multicolumn{4}{|c|}{$\operatorname{AFE}\left(\mathrm{g} \mathrm{cm}^{-2}\right)$} \\
\hline Cayman & - & - & - & - & $191^{b}$ & $201^{a}$ & $177^{c}$ & $190^{a}$ \\
\hline Cobra & - & - & - & - & $190^{b c}$ & $192^{a b}$ & $181^{b c}$ & $188^{a b}$ \\
\hline Mulato II & - & - & - & - & $196^{a}$ & $191^{b}$ & $184^{a b}$ & $190^{a}$ \\
\hline Insurgente & - & - & - & - & $175^{d}$ & $174^{c}$ & $170^{d}$ & $173^{c}$ \\
\hline $\mathrm{H}-17$ & - & - & - & - & $187^{c}$ & $187^{b}$ & $187^{a}$ & $187^{b}$ \\
\hline Promedio & - & - & - & - & $188^{A}$ & $189^{A}$ & $180^{B}$ & \\
\hline
\end{tabular}

vares Cayman y Mulato II presentaron la mayor acumulación de MSh (8.44 y 8.49 t MS ha $^{-1}$, respectivamente); mientras que, a las 8 semanas la mayor acumulación ocurrió en los cultivares Mulato II, Insurgente y Cobra con promedio de $8 \mathrm{t} \mathrm{MS} \mathrm{ha}{ }^{-1}$. Se encontró efecto de interacción $(\mathrm{p} \leq 0.05)$ entre los cultivares Cayman, Cobra e Insurgente y la edad de rebrote. Durante la época de menor precipitación, todos los cultivares de Urochloa presentaron mayor acumulación de MSh con valores de 1.00 a $1.08 \mathrm{t}$ MS ha ${ }^{-1}$, en comparación con el cultivar H-17 (0.79 t MS ha ${ }^{-1}$ ).

La mayor acumulación de MSh en los cultivares de Urochloa, en comparación con Pennisetum ciliare cv. H-17, se atribuyó a que los cultivares de Urochloa presentan mayores tasas de elongación de hojas y diferente distribución de la materia seca en la planta (Dias-Filho y Carvalho 2000). Al respecto, Guenni et al. (2005) reportaron para $U$. decumbens y $U$. brizantha tasas de elongación en hojas de 2.5 y $3.5 \mathrm{~cm} \mathrm{~d}^{-1}$, respectivamente. Así mismo, Bernal et al. (2016) mencionan que los híbridos Cayman y Mulato II, son cultivares que producen mayor cantidad de hojas y, de acuerdo con Cruz et al. (2011b), la hoja es el componente que más aporta al rendimiento. También se ha indicado que los factores ambientales, principalmente la precipitación y temperatura, y el manejo de la pradera, determinan la dinámica en la acumulación de materia seca de hojas y tallos. Al respecto se observó que durante la época de máxima precipitación se tuvo mayor diferenciación de los órganos de planta (hojas y tallos), lo cual se debe al recambio de tejidos como resultado del crecimiento activo (Castro et 


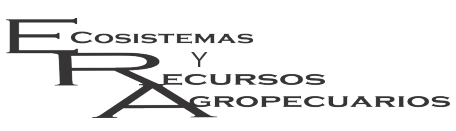

al. 2013). En cambio, durante la época de menor precipitación, se registró menor tasa de crecimiento $y$, en consecuencia, menor acumulación de materia seca. La mayor acumulación de materia seca de hoja obtenida a las 4 y 6 semanas de rebrote, indica mejor calidad del forraje cosechado, ya que, a mayor proporción de hojas, el contenido de proteína cruda en la materia seca total es mayor (Garay et al. 2017b).

\section{Acumulación de materia seca de tallo (MSt)}

Se encontró diferencia entre cultivares y edad de rebrote para la MSt $(p \leq 0.05)$. Durante la época de mayor precipitación se observó que el cultivar $\mathrm{H}-17$ presentó la mayor acumulación de tallos, con un valor de $1.5 \mathrm{t} \mathrm{MS} \mathrm{ha}^{-1}$, seguido de Cobra (0.9 $\mathrm{t} \mathrm{MS} \mathrm{ha}^{-1}$ ); mientras que, independientemente de la edad de rebrote, los cultivares Mulato II, Insurgente y Cayman presentaron la menor acumulación de tallo (Tabla 1). Se observó que la acumulación de materia seca de tallo se incrementó conforme aumentó la edad de rebrote, con valores de $0.44,0.73$ y $1.06 \mathrm{t} \mathrm{MS} \mathrm{ha}^{-1}$, a las 4, 6 y 8 semanas, respectivamente. Durante la época de menor precipitación, en ningún cultivar se presentó acumulación de materia seca de tallo (Tabla 1 ).

Las diferencias entre cultivares en la acumulación de MSt se atribuyó al ciclo fenológico más corto que presentan algunos cultivares (Bernal et al. 2016), como los cultivares $\mathrm{H}-17$ y Cobra, así como por las distintas tasas de elongación de los tallos. Al respecto, Paciullo et al. (2011), reportaron tasas de elongación para $U$. decumbens, $U$. brizantha cv. Xaraés y $U$. brizantha cv. Insurgente de $1.2,0.8$ y $0.6 \mathrm{~mm}$ tallo ${ }^{-1} \mathrm{~d}^{-1}$, respectivamente. Durante el periodo de menor precipitación, se obtuvieron los menores rendimientos de MSt, como consecuencia del estrés hídrico por deficiencia de humedad, que limitó el crecimiento del tallo (Cruz et al. 2011a), lo que se reflejo en la disminución del crecimiento ade las plantas (Cab et al. 2008). De igual manera, la acumulación de materia seca de tallos incrementó, conforme aumentó la edad de rebrote; lo cual podría indicar que, cosechar cada 8 semanas de rebrote, durante la época de mayor
Garay-Martínez et al. Comportamiento forrajero de pasto Urochloa Ecosist. Recur. Agropec. 5(15):573-581,2018

precipitación, se obtiene forraje de menor calidad, ya que al aumentar la cantidad de tallos disminuye la digestibilidad y, en consecuencia, el consumo de forraje por los animales (Cavalcante et al. 2014).

\section{Área foliar especifica (AFE)}

Se encontraron diferencias entre cultivares y edad de rebrote para la variable AFE $(p \leq 0.05)$. Durante la época de mayor precipitación, el valor más alto $\left(207 \mathrm{~cm}^{2} \mathrm{~g}^{-1}\right)$ se obtuvo con el cultivar Cayman, seguido de los cultivares Cobra y Mulato II con promedio de $191 \mathrm{~cm}^{2} \mathrm{~g}^{-1}$; mientras que los cultivares Insurgente y $\mathrm{H}-17$ presentaron la menor AFE, con valores de 180 y $176 \mathrm{~cm}^{2} \mathrm{~g}^{-1}$, respectivamente (Tabla 1). Durante la época de menor precipitación la mayor AFE se obtuvo con los cultivares Cayman, Mulato II y Cobra, con valores entre 188 y $190 \mathrm{~cm}^{2}$ $\mathrm{g}^{-1}$; mientras que el cultivar Insurgente registró la menor AFE $\left(173 \mathrm{~cm}^{2} \mathrm{~g}^{-1}\right)$. En ambas épocas la edad de rebrote afectó $(\mathrm{p} \leq 0.05)$ el AFE, ya que se presentó una disminución en los valores a medida que se incrementó la edad de rebrote.

Las diferencias observadas en AFE en los cultivares evaluados puede deberse a que los cultivares de Urochloa presentan variaciones en la morfología y estructura de la hoja. Al respecto, Baruch y Guenni (2007) reportaron para U. decumbens y U. brizantha, a las cuatro semanas de edad con promedios de 300 y $270 \mathrm{~cm}^{2} \mathrm{~g}^{-1}$, respectivamente. Estos valores son superiores a los encontrados en el presente estudio. La diferencia de los valores obtenidos en AFE entre edades de rebrote se atribuye al aumento en altura conforme avanzó la edad de la planta, lo que ocasionó mayor proporción de hojas maduras y de mayor espesor, en comparación con las hojas jóvenes (Yasuoka et al. 2018). Lo que ocasionó disminución en los valores de AFE en edades mayores de rebrote. Al respecto se sabe que los pastos con mayor AFE tienen hojas de menor espesor y concentraciones altas de nitrógeno (Pérez et al. 2004), las cuales son consumidas por los animales en mayor cantidad (Zheng et al. 2014). Por tanto, los híbridos de Urochloa evaluados, podrían tener mayor contenido de proteína y mejor aceptación por los animales, ya que presentaron los valores más altos 
de AFE, en comparación con los cultivares Insur-

gente y Pennisetum ciliare cv. H-17.

Todos los cultivares de Urochloa evaluados presentaron alta acumulación de materia seca total, en comparación con Pennisetum ciliare cv. H-17; aun cuando no se encontraron en un ambiente para el cual fueron mejorados. Los cultivares Mulato II y Cayman, presentaron mayor acumulación de materia seca total, con promedio de $10 \mathrm{t} \mathrm{MS} \mathrm{ha}{ }^{-1}$; así como los valores mayores de materia seca de hoja y área foliar específica. Independientemente del cultivar, para un mejor aprovechamiento del forraje, durante la época de mayor precipitación se debe cosechar entre la cuarta y sexta semana de rebrote; mientras que en la época de menor precipitación a las ocho semanas.

\section{AGRADECIMIENTOS}

Al Consejo Nacional de Ciencia y Tecnología (CONACYT) por la beca (CVU/Becario: 425135/266100) del primer autor. Nuestro agradecimiento al Grupo Papalotla y la Facultad de Ingeniería y Ciencias de la Universidad Autónoma de Tamaulipas por las facilidades proporcionadas para la investigación.

\section{LITERATURA CITADA}

Arroyave C, Tolrà R, Thuy T, Barceló J, Poschenrieder C (2013) Differential aluminum resistance in Brachiaria species. Environmental and Experimental Botany 89: 11-18.

Avellaneda C, Cabezas JF, Quintana G, Luna R, Montañez O, Espinoza I, et al. (2008) Comportamiento agronómico y composición química de tres variedades de Brachiaria en diferentes edades de cosecha. Ciencia y Tecnología 1: 87-94.

Bernal A, Velázquez VH, Ruíz R, Quero AR, Pizarro EA (2016) Potencial productivo en tres híbridos de Urochloa. En: Cantú A, González EA, López R, Ávila JM (ed). III Congreso Mundial de Ganadería Tropical 2016. Tampico, Tamaulipas, México. pp: 100-104.

Baruch Z, Guenni O (2007) Irradiance and defoliation effects in three species of the forage grass Brachiaria. Tropical Grasslands 41: 269-276.

Cab F, Enríquez J, Pérez J, Hernández A, Herrera J, Ortega E, et al. (2008) Potencial productivo de tres especies de Brachiaria en monocultivo y asociadas con Arachis pintoi en Isla, Veracruz. Técnica Pecuaria en México 46: 317-332.

Cardoso JA, Pineda P, Jiménez JC, Vergara MF, Rao IM (2015) Contrasting strategies to cope with drought conditions by two tropical forage $\mathrm{C} 4$ grasses. AoB Plants 7: 1-15.

Castro R, Hernández A, Ramírez O, Aguilar G, Enríquez JF, Mendoza SI (2013) Crecimiento en longitud foliar y dinámica de población de tallos de cinco asociaciones de gramíneas y leguminosa bajo pastoreo. Revista Mexicana de Ciencias Pecuarias 4: 201-215.

Cavalcante ACR, Araújo JF, Carneiro MS, Souza HA, Tonucci RG, Rogerio MCP, et al. (2014) Potential use of tropical grass for deferment in Semi-Arid Region. American Journal of Plant Sciences 5: 907-914.

CONAGUA (2010) Normales climatológicas por Estado. Comisión Nacional de Agua (CONAGUA)- Sistema Meteorológico Nacional (SMN). http://smn.cna.gob.mx/index.php?option=com_content\&view=article \&id=42\&ltemid=75. Fecha de consulta: 8 de agosto de 2017.

Cruz A, Hernández A, Enríquez J, Gómez A, Ortega E, Maldonado N (2011a) Producción de forraje y composición morfológica del pasto Mulato (Brachiaria híbrido 36061) sometido a diferentes regímenes de pastoreo. Revista Mexicana de Ciencias Pecuarias 2: 429-443. 
Cruz PI, Hernández A, Enríquez JF, Mendoza SI, Quero AR, Joaquín BM (2011b). Desempeño agronómico de genotipos de Brachiaria humidicola (Rendle) Schweickt en el trópico húmedo de México. Revista Fitotecnia Mexicana 34: 123-131.

Cruz-Hernández A, Hernández-Garay A, Aranda-Ibañez E, Chay-Canul AJ, Márquez-Quiroz C, Rojas-García AR, et al. (2017) Nutritive value of Mulato Grass under different grazing strategies. Ecosistemas y Recursos Agropecuarios 4: 65-72.

Donaghy DJ, Turner LR, Adamczewski KA (2008) Effect of defoliation management on water-soluble carbohydrate energy reserves, dry matter yields, and herbage quality of tall fescue. Agronomy Journal 100: 122-127.

Dias-Filho MB, Carvalho CJR (2000) Physiological and morphological responses of Brachiaria spp. to flooding. Pesquisa Agropecuária Brasileira 35: 1959-1966.

Dourado RL, De Souza AL, Zanine AM, Toral FLB, Ferreira DJ, De Abreu JG (2015) Structural and production characteristics of Piatã grass forage submitted to levels of nitrogen. American Journal of Plant Sciences 6: 693-701.

Durán N, Ruiz JA, González DR, Núñez G, Padilla FJ, Contreras SH (2011) Temperaturas cardinales de desarrollo en la etapa siembra-emergencia de 11 pastos forrajeros. Revista Mexicana de Ciencias Pecuarias 2: $347-357$.

Garay JR, Joaquín S, Estrada B, Martínez JC, Hernández J, Limas AG, et al. (2017a) Rendimiento de forraje de Pennisetum ciliare cv. H-17 a tres edades de rebrote en Güémez, Tamaulipas, México. En: Yamasaki A, Yamasaki L, Yong G, Macias GP, León H, Pérez EJ, Sánchez J (ed). XLIV Reunión Científica AMPA 2017, Clima y Ganadería: Productividad Sustentable. Tuxtla Gutiérrez, Chiapas, México. pp: 470-475.

Garay JR, Joaquín S, Zárate P, Ibarra MA, Martínez JC, González RP, et al. (2017b) Dry matter accumulation and crude protein concentration in Brachiaria spp. cultivars in the humid tropics of Ecuador. Tropical Grasslands-Forrajes Tropicales 5: 66-76.

Gómez E, Díaz H, Saldívar A, Briones F, Vargas V, Grant W (2007) Patrón de crecimiento de pasto buffel [Pennisetum ciliare L. (Link.) Sin. Cenchrus ciliaris L.] en Tamaulipas, México. Técnica Pecuaria en México 45: 1-17.

Guenni O, Gil JL, Guedez Y (2005) Growth, forage yield and light interception and use by stands of five Brachiaria species in a tropical environment. Tropical Grasslands 39: 42-53.

INEGI (2015) Anuario estadístico y geográfico de Tamaulipas 2015. Instituto Nacional de Estadística y Geografía. México. 521p.

Lara C, Oviedo LE, Betancur CA (2010) Efecto de la época de corte sobre la composición química y degradabilidad ruminal del pasto Dichanthium aristatum (Angleton). Zootecnia Tropical 28: 275-281.

Maass BL, Midega CAO, Mutimura M, Rahetlah VB, Salgado P, Kabirizi JM, et al. (2015) Homecoming of Brachiaria: Improved hybrids prove useful for african animal agriculture. East African Agricultural and Forestry Journal 81: 71-78.

Miles JW (2006) Mejoramiento genético en Brachiaria: objetivos, estrategias, logros y proyecciones. Pasturas Tropicales 28: 26-30.

Morales-Bautista C, Domínguez-Rodríguez V, Adams RH (2011) Estudio cinético del intercambio catiónico con $\mathrm{Ca}(\mathrm{OH})_{2}$ y evaluación de la fertilidad en un suelo arcilloso contaminado con aguas congénitas. Bioagro 23: 129-134. 
Paciullo D, Fernandes P, Gomide C, De Castro D, Sobrinho F, Augusto C, et al. (2011) The growth dynamics in Brachiaria species according to nitrogen dose and shade. Revista Brasileira de Zootecnia 40: 270-276.

Pérez JA, García E, Enríquez JF, Quero AR, Pérez J, Hernández A (2004) Análisis de crecimiento, área foliar específica y concentración de nitrógeno en hojas de pasto "mulato" (Brachiaria híbrido, cv.). Técnica Pecuaria en México 42: 447-458.

Pizarro EA, Ruíz RR, Ávila F, López A (2010) Informe anual. Departamento de Investigación y Desarrollo. Grupo Papalotla. México. 57p.

Pizarro E, Hare M, Mutimura M, Changjun B (2013) Brachiaria hybrids: potential, forage use and seed yield. Tropical Grasslands-Forrajes Tropicales 1: 31-35.

SAGARPA (2010) Informe de evaluación externa estatal de los programas en coejercicio. Diagnóstico Sectorial 2009. SAGARPA y Gobierno del Estado de Tamaulipas. México. 140p.

SAS (2004) SAS/STAT ${ }^{\circledR} 9.1$ user's guide. SAS Publishing. SAS Institute Inc., NC, USA. 5121p.

SIAP (2014) Cierre de la producción agrícola por cultivo. Anuario Estadístico de la Producción Agrícola. http://www.siap.gob.mx/cierre-de-la-produccion-agricola-por-cultivo/.Fecha de consulta 8 de agosto de 2017.

Vargas TV, Hernández RME, Gutiérrez LJ, Plácido DCJ, Jiménez CA (2007) Clasificación climática del Estado de Tamaulipas, México. CienciaUAT 2: 15-19.

Yasuoka JI, Pedreira CGS, Da Silva VJ, Alonso MP, Da Silva LS, Gomes FJ (2018) Canopy height and N affect herbage accumulation and the relative contribution of leaf categories to photosynthesis of grazed brachiariagrass pastures. Grass and Forage Science 73: 182-193.

Zheng SX, Li WH, Lan ZC, Ren HY, Wang KB, Bai YF (2014) Testing functional trait-based mechanisms underpinning plant responses to grazing and linkages to ecosystem functioning in grasslands. Biogeosciences Discuss 11: 13157-13186. 
